\title{
H $\infty$ Robust Control for Chaotic Motion of the Fractional-Order D-PMSG via the PDC Approach
}

\author{
Li Yang $\mathbb{D}^{1,2}$ Zijie Shen $\mathbb{D}^{1},{ }^{3}$ Weitao Sheng, ${ }^{2}$ and Tianmin Huang ${ }^{1}$ \\ ${ }^{1}$ School of Electrical Engineering, Southwest Jiaotong University, Chengdu 610031, China \\ ${ }^{2}$ Electrical \& Information Engineering Department, Sichuan Engineering Technical College, Deyang 618000, China \\ ${ }^{3}$ School of Information Science and Engineering, Chengdu University, Chengdu 610106, China
}

Correspondence should be addressed to Li Yang; yangli@scetc.edu.cn

Received 3 June 2021; Revised 6 August 2021; Accepted 17 August 2021; Published 6 September 2021

Academic Editor: Tongqian Zhang

Copyright (c) 2021 Li Yang et al. This is an open access article distributed under the Creative Commons Attribution License, which permits unrestricted use, distribution, and reproduction in any medium, provided the original work is properly cited.

\begin{abstract}
The stability analysis and controller design problems for the fractional-order (FO) direct-drive permanent magnet synchronous generator (FOD-PMSG) wind turbine with parameter uncertainties and external disturbance are addressed. Takagi-Sugeno (T-S) model is used to approximate nonlinearities, and the parallel distributed compensation (PDC) technique is employed to construct the fuzzy state feedback controller. In order to suppress the external disturbance more effectively, the global Mittag-Leffler stability definition satisfying the Ho performance index is proposed for the first time. Using the FO Lyapunov direct method, applying the Cauchy matrix inequality (CMI), and combining with the Schur complement lemma, the sufficient conditions of Mittag-Leffler stability meeting the Ho performance index are given in the form of linear matrix inequalities (LMIs). Simulation results clearly show that the proposed control scheme can make the system get rid of the chaotic state quickly and have strong robustness under parameter uncertainties and external disturbance varying randomly.
\end{abstract}

\section{Introduction}

D-PMSG is of great interest for large offshore wind farms with rich wind energy resources, less land occupation, and low water consumption due to the outstanding advantages of small mechanical loss, low maintenance cost, high reliability, strong grid connection adaptability, and simple structure $[1,2]$. Wind turbine systems (WTSs) have the characteristics of randomness of wind energy, strong coupling nonlinear complex characteristics, and bad working environment, which lead to the inevitable changes of system parameters and external disturbances in the process of the system operation and then make WTSs show chaotic phenomenon under certain working conditions. This will seriously affect the stable operation of WTSs, have a greater impact on the grid, and make power quality worse. The nonlinear dynamic behaviour, chaos generation mechanism, and chaotic control of the D-PMSG have recently received wide attention and become an active research area.

Recently, numerous research results have been obtained in the analysis and control of chaos characteristics of the permanent magnet synchronous motor (PMSM) $[3,4]$. It is found for the first time that PMSM-based wind energy conversion systems (WECs) have chaotic operation state [5]. Then, dynamic characteristic analysis and chaotic control of the WECS are favoured by many scholars [6-8]. The chaotic motion in D-PMSG-based WTSs under certain parameters is investigated, and a new action-dependent heuristic dynamic programming (ADHDP) method based on the Cloud radial basis function (RBF) neural network is proposed to track the point of maximum wind power which can suppress chaotic motion and track the point of maximum power stably [6]. The chaos mathematical model of the D-PMSG was established in PSCAD/EMTDC software, and the chaos phenomenon caused by the change of wind speed was validated [7]. It is notable that intensive efforts have been devoted to the research of the fractional calculus in modelling and characterizing accurate dynamical properties of numerous physical phenomena in nature and control system design recently [9-12]. Following this trend, some remarkable research results show that the motor system and the power system have FO characteristics [13-15]. Fractional 
calculus is used to establish the mathematical model of the motor [16]. The chaotic characteristics of the FO-PMSM are studied by the three-phase diagram, Lyapunov exponent spectrum, and Poincare mapping, and the FO model is realized by the electronic circuit [17]. The FO controller based on the T-S model is designed for a doubly fed wind turbine [18].

Up till the present moment, it is still a challenging problem to design the suitable controller for the nonlinear WTSs. It is clear from the literature studies that numerous control algorithms on nonlinear forms of WTSs are reported such as intelligent control [19], adaptive control [20], sliding mode control (SMC) [21], and fuzzy control [22-25]. In [19], an integrated intelligent automatic control system is proposed to increase the extracting energy of the doubly fed induction generator-based WECs. In [20], a robust MPPT control scheme for grid-connected PMSG-based WTSs using perturbation observation via nonlinear adaptive control is presented. In [21], an improved SMC is proposed for PMSG-based WECs. To overcome the controlling issues of nonlinear systems, T-S fuzzy model [22] can transform the nonlinear model into the linear submodel without affecting the properties of the system. In the view of tremendous results on the T-S fuzzy model, an integer-/ fractional-order T-S fuzzy system has received much attention among the researchers [23-28]. In particular, in [25], a PDC fuzzy controller has been proposed for a class of FO nonlinear systems (FONSs) with parameter uncertainties and proved its effectiveness with the help of numerical simulation. In [28], an Ho robust state feedback controller for uncertain T-S fuzzy systems by a nonmonotonic Lyapunov function has been proposed.

In addition, it is necessary to concentrate on how to control chaotic behaviour of PMSG-based WTSs under complex working conditions and design a suitable control scheme. A disturbance observer-based integral fuzzy SMC methodology for WTSs with a nonlinear PMSG is proposed using the T-S fuzzy model [29]. A novel FO fuzzy logic control for PMSG-based WTSs is presented [30]. In [31], an improved augmented T-S fuzzy control scheme is applied to the system of converting wind turbine energy. In [32], the relationship between the FO system and integer-order system has been revealed, and a delayed feedback control scheme is introduced to control the FO-PMSM model of a wind turbine. The chaotic characteristics in the FOD-PMSG model are detected, and a novel predictive control scheme in the FO sense is designed to suppress chaos [33]. The FO characteristic of the D-PMSG wind turbine is researched, and an adaptive fuzzy sliding mode controller is designed based on the T-S model [34], but the system parameter uncertainties are not considered. In [35], an observer-based event-triggered (ET) fuzzy integral SMC for a nonlinear chaotic PMSG is designed.

Against external disturbances, how to construct an appropriate Lyapunov function and what methods are used to ensure the stable Ho control performance of the closedloop system in terms of solvable LMIs have received much attention among the researchers. In [28], through the nonmonotonic Lyapunov function approach, based on the terms of LMIs, the sufficient conditions for the existence of a robust state feedback Ho controller which guarantees the system stability and a prescribed Ho performance are given. Huong and Thuan [36] first presented a mixed Ho and passive control for a class of control FONSs. In [35], a suitable Lyapunov-Krasovskii function has been constructed to ensure the stable Ho performance of the system in terms of solvable LMIs through the convex matrix inequality method. In order to expand the system stability region and improve the stable performance of the WTSs, as an improvement, the present research aims to design the suitable controller scheme for the D-PMSG model with parameter uncertainties and external interference in the fractional domain instead of traditional controller schemes. Motivated by the aforementioned progress, we design a fuzzy state feedback Ho robust controller that is determined through a quadratic Lyapunov function and solving a set of LMIs. In this paper, the main objective of this study is to address the chaos control problem of FOD-PMSG-based WTSs with uncertain parameters and external disturbances. During the controller design process, T-S model is applied to approximate the nonlinearities, and the fuzzy state feedback Ho robust controllers are constructed via PDC technology. The factors that need to be solved in this paper are how to derive the global Mittag-Leffler stability conditions based on the LMI form by the fractional Lyapunov direct method.

Bearing the above problems, this paper is organized as follows: Section 2 contains the necessary preliminaries and definitions. Section 3 describes the formulation of the T-S fuzzy FOD-PMSG model from the nonlinear integer-order D-PMSG via membership rules. Section 4 contains the derivation of the main results. Numerical simulations of the D-PMSG are performed in Section 5. This paper gives the concluding comments and discussion in Section 6.

\section{Preliminaries and Problem Formulation}

In this section, some basic theorems, definitions, and lemmas of the FO system will be introduced. The initial value of the Caputo fractional derivative has clear physical meaning and is widely used in engineering practice.

Definition 1 (see [37]). The Caputo fractional derivative of $\alpha>0$ for a continuous function $f(t)$ is defined as follows:

$$
D_{t}^{\alpha} f(t)=\frac{1}{\Gamma(n-\alpha)} \int_{t_{0}}^{t} \frac{f^{(n)}(\tau)}{(t-\tau)^{1+\alpha-n}} \mathrm{~d} \tau,
$$

where $D_{t}^{\alpha}$ is the Caputo fractional derivative of $\alpha$-order, $\alpha \in \mathrm{R}$, $n=[\alpha]$, and $\Gamma()$ is the Gamma function.

Definition 2 (see [37]). The Caputo fractional integral of $\alpha>0$ for a continuous function $f(t)$ is defined as

$$
I_{t}^{\alpha} f(t)=\frac{1}{\Gamma(\alpha)} \int_{t_{0}}^{t}(t-\tau)^{\alpha-1} f(\tau) \mathrm{d} \tau,
$$

where It $\alpha$ is the Caputo fractional integral of $\alpha$-order. 
Remark 1. $D_{t}^{\alpha}={ }_{0 t}^{\alpha} D$ represents the $\alpha$-order differential when time is from 0 to $t$, and $I_{t}^{\alpha}={ }_{0 t}^{\alpha} I$ is the $\alpha$-order integral.

Property 1 (see [38]). If $f(t)$ is a continuous function, $n-1<\alpha<n, n-1<\beta-\alpha<n$, and $\alpha, \beta \in \mathrm{R}+$, then we have

$$
I_{t}^{\alpha} D_{t}^{\beta} f(t)=D_{t}^{\beta-\alpha} f(t) \text {. }
$$

Definition 3 (see [39]). For a FO nonlinear dynamic system $D_{t}^{\alpha} x(t)=f(t, x)$, the constant $x_{e}$ is an equilibrium point of the equation $f\left(t, x_{e}\right)=D_{t}^{\alpha} x_{e}$.

In general, it is assumed that $x_{e}=0$ is the equilibrium point of the FO system.

Definition 4 (see [39]). The autonomous fractional differential system can be described as follows:

$$
D_{t}^{\alpha} x(t)=f(t, x)=A x,
$$

wherein $\alpha_{i} \in(0,1](i=1,2, \ldots, n), D_{t}^{\alpha}=\left[D_{t}^{\alpha_{1}}, D_{t}^{\alpha}, \ldots, D_{t}^{\alpha_{n}}\right]$, $A \in R^{n \times \mathrm{n}}$, and $f() \in R^{n}$. There is $\alpha_{i}=p_{i} / q_{i}$ in equation (4), and $N$ is the least common multiple of all $q_{i}$. If $x_{e}$ makes the equation $f\left(t, x_{e}\right)=D_{t}^{\alpha} x_{e}$, then $x_{e} \in R^{n}$ is the equilibrium point of system (4).

Lemma 1 (see [40]). FO Lyapunov direct method). Let $x(t)=$ 0 be the equilibrium point of the system $D_{t}^{\beta} x(t)=f(t, x)$ and $\Omega \subset R^{n}$ be a region containing the origin. If there are any normal numbers $l_{1}, l_{2}, l_{3}, a$, and $b$ and continuously differentiable functions $V(t, x)$ and inequalities (5) and (6) hold,

$$
\begin{aligned}
l_{1}\|x(t)\|^{a} & \leq V(t, x(t)) \leq l_{2}\|x(t)\|^{b}, \\
D_{t}^{\alpha} V(t, x(t)) & \leq-l_{3}\|x(t)\|^{a b},
\end{aligned}
$$

wherein $t>0, x \in \Omega, \alpha \in(0,1], V(t, x):[0, \infty] \times O \longrightarrow R$ is locally Lipschitz continuous on $\Omega \subset R^{n}$, then the equation $x=0$ is Mittag-Leffler stable.

Lemma 2 (see [41]). If $x(t) \in R^{n}$ is a continuous differentiable function and $P \in R^{n \times n}$ is any positive definite matrix, the following condition holds:

$$
\frac{1}{2} D_{t}^{\alpha} x^{T}(t) P x(t) \leq x^{T}(t) P D_{t}^{\alpha} x(t) .
$$

Lemma 3 (see [42]). Given matrices $M$ and $N$ of appropriate dimensions, for any $\Delta F(t)$ satisfying $\Delta F^{T}(t) \Delta F(t) \leq I$, if and only if there exists $\zeta^{\prime}>0, M \Delta F$ (t) $N+N \quad T \Delta F^{T}$ (t) $M^{T} \leq \zeta M M^{T}+\zeta^{-1} N^{T} N$ holds.

Lemma 4 (see [43]). For a given symmetric matrix $S$, the following inequalities are equivalent:

$$
\begin{aligned}
& \text { (1) } S=\left[\begin{array}{ll}
S_{11} & S_{12} \\
S_{21} & S_{22}
\end{array}\right]=\left[\begin{array}{ll}
S_{11} & S_{12} \\
S_{12}^{T} & S_{22}
\end{array}\right]<0 \\
& \text { (2) } S_{11}<0, S_{22}-S_{12}^{T} S_{11}^{-1} S_{12}<0 \\
& \text { (3) } S_{22}<0, S_{11}-S_{12} S_{11}^{-1} S_{12}^{T}<0
\end{aligned}
$$

Lemma 5 (see [42]). For any given vectors $m, n \in R^{n}$, the following inequality holds:

$$
2 m^{T} n \leq \eta m^{T} m+\eta^{-1} n^{T} n, \quad \forall \eta>0 .
$$

Lemma 6 (see [39]). For $x(t) \in C^{1}\left[0, T_{0}\right]$ with $T_{0}>0$, we can have

$$
\begin{aligned}
& I_{t}^{\alpha} D_{t}^{\alpha} x(t)=x(t)-x(0), \\
& D_{t}^{\alpha} I_{t}^{\alpha} x(t)=x(t)
\end{aligned}
$$

Lemma 7 (see [44]). The necessary conditions for the existence of chaos in autonomous system (4) are as follows:

(1) For the commensurate FO systems with $\alpha_{1}=\alpha_{2}=\ldots=$ $\alpha_{n}=\alpha$, system (4) is asymptotically stable if and only if $\min \left\{\left|\arg \left(\lambda_{i}\right)\right|\right\}>\alpha \pi / 2$ is satisfied for all eigenvalues $\lambda_{i}$ $(i=1,2, \ldots, n)$ of Jacobian matrix $J=\partial f / \partial x \mid x e$

(2) For the incommensurate FO systems with $\alpha_{1} \neq \alpha_{2} \neq \ldots$ $\neq \alpha_{n}$, system (4) is also asymptotically stable if and only if $\min \left\{\left|\arg \left(\lambda_{i}\right)\right|\right\}>\pi / 2 N$ is satisfied for all eigenvalues $\lambda_{i}\left[i=1,2, \ldots, N\left(\alpha_{1}+\alpha_{2}+\ldots+\alpha_{n}\right)\right]$ in det $\left(\operatorname{diag}\left(\left[\lambda^{N \alpha_{1}}, \lambda^{N \alpha_{2}}, \ldots, \lambda^{N \alpha_{n}}\right]\right)-J\right)=0$

\section{D-PMSG Wind Turbine Model}

3.1. Integer-Order D-PMSG Wind Turbine Model. The dimensionless Lorenz mathematical model of the D-PMSG with uncertain parameters is obtained by linear affine transformation and time-scale transformation $[5,45]$ :

$$
\left\{\dot{\vec{i}}_{d}=-\widetilde{i}_{d}+\widetilde{i}_{q} \widetilde{w}_{g}+\widetilde{u}_{d}, \dot{\vec{i}}_{q}=-\widetilde{i}_{q}-\widetilde{i}_{d} \widetilde{w}_{g}+\mu \widetilde{w}_{g}+\widetilde{u}_{q}, \dot{\tilde{w}}_{g}=\sigma\left(\widetilde{i}_{q}-\widetilde{w}_{g}\right)-\widetilde{T}_{m},\right.
$$

wherein $\widetilde{i}_{d}, \widetilde{i}_{q}$, and $\widetilde{w}_{g}$ are the transformed direct axis, quadrature current component, and generator speed, respectively; $\widetilde{u}_{d}, \widetilde{u}_{q}$, and $\widetilde{T}_{m}$ are the transformed $d$-axis, $q$-axis voltage component, and wind turbine torque, respectively; and $\sigma$ and $\mu$ are the system parameters with $\sigma \in\left[\sigma_{1}, \sigma_{2}\right]$ and $\mu \in\left[\mu_{1}, \mu_{2}\right]$.
In chaos model (10), it can be found that $\sigma$ and $\mu$ only depend on the system parameters. The system parameters studied in this paper are shown in Table 1.

In order to verify the chaotic characteristics for the D-PMSG, the simulation results are shown in Figure 1 when 
TABLE 1: The descriptions of the parameters used in model (10) [5].

\begin{tabular}{lcccc}
\hline Parameter & Symbol & Unit & Value & Remark \\
\hline$d$-axis inductance & $L_{d}$ & $\mathrm{mH}$ & 2.7 & 2.7 \\
$q$-axis inductance & $L_{q}$ & $\mathrm{mH}$ & 1.14 & Varies \\
Armature resistance & $\mathrm{Rs}$ & $\mathrm{mWb}$ & 212 & - \\
Permanent magnet flux & $\Psi \mathrm{f}$ & $\mathrm{kg} \cdot \mathrm{m}^{2}$ & 0.089 & Varies \\
Moment of inertia & $J_{e d}$ & $\mathrm{~N} \cdot \mathrm{ms}$ & - \\
Friction constant & $B_{m}$ & - & 0.1204 & - \\
Number of pole pairs & $n_{p}$ & - & 17 \\
\hline
\end{tabular}
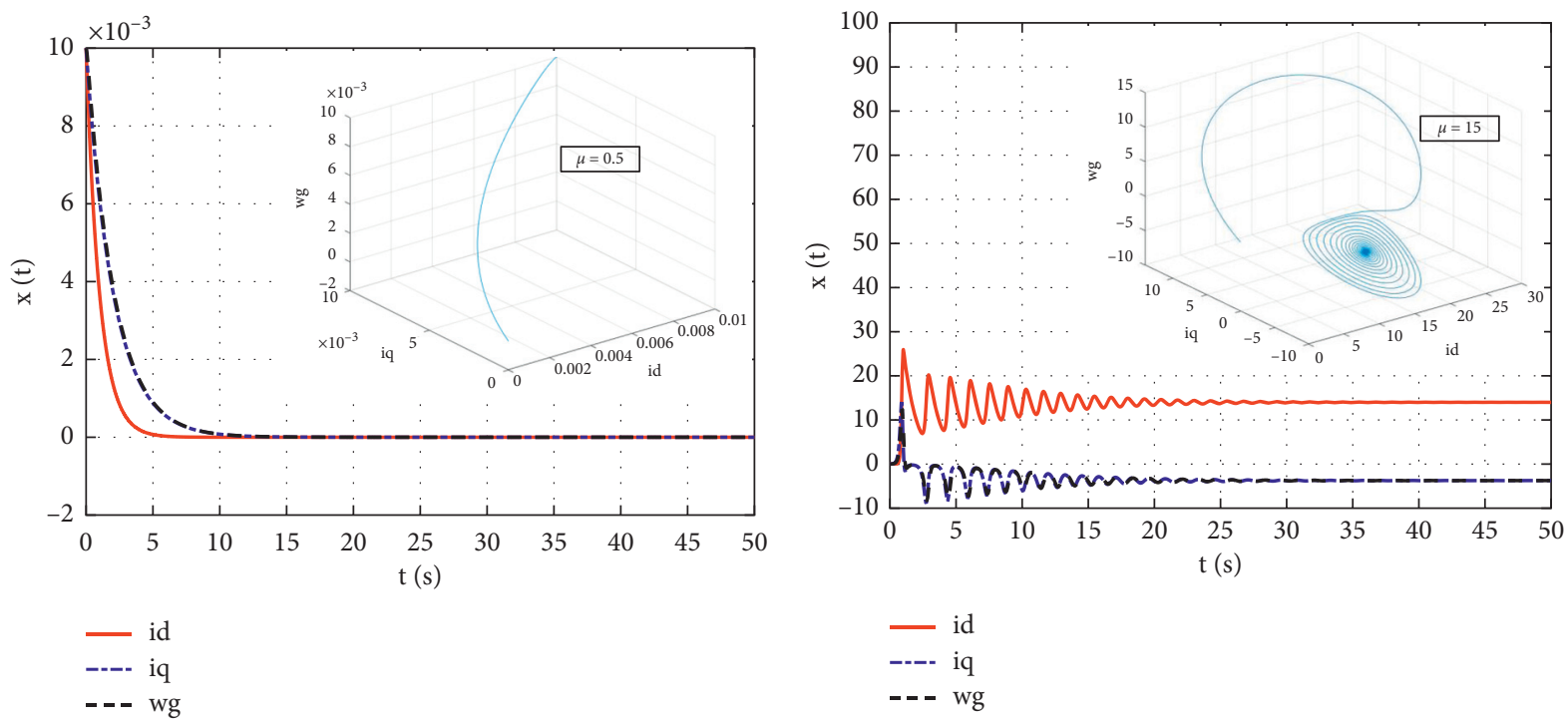

(a)

(b)

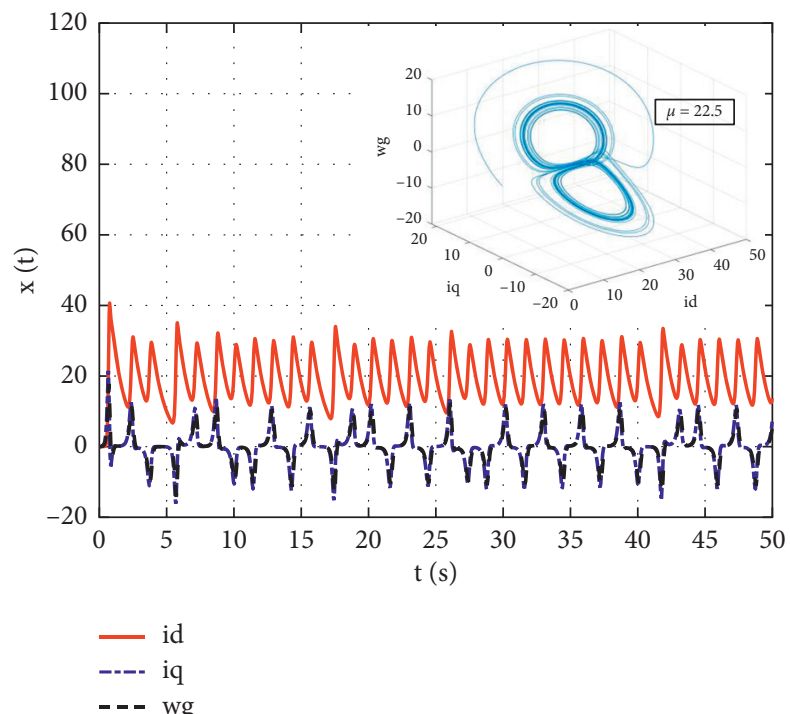

(c)

FIgURE 1: The system chaotic characteristics with $\mu$ varying. (a) $\mu=0.5$. (b) $\mu=15$. (c) $\mu=22.5$.

the system parameters are selected as $\sigma=16$ and $\mu=0.5$, $\mu=15$, and $\mu=22.5$, respectively.

It can be seen from Figure 1 (a) that when $\mu<1$, the system has only one stable equilibrium point and is in a stable state. Figure $1(\mathrm{~b})$ shows the system with $\mu=15$ is also stable. Although the stable speed is relatively slow, the system can also reach a stable state with the further increase of time. Figure 1(c) shows the system exhibits chaotic action for $\mu=22.5$. By the above analysis, Figure 1 shows different dynamic characteristics under different parameter selection.

The chaotic characteristics for the D-PMSG are studied by taking $L_{d}=L_{q}=14.38 \mathrm{H}, J_{e d}=0.0089 \mathrm{~kg} \cdot \mathrm{m}^{2}, R_{s}=1.14 \Omega$, $B_{m}=0.1204 \mathrm{Nms}$, the system parameters $\sigma=16$ and $\mu=20$, and external input parameters $\widetilde{u}_{d}=-0.542, \widetilde{u}_{q}=0.824$, and 
$\widetilde{T}_{m}=1.16$. Figure 2(a) shows that the state of the D-PMSG tends to be stable, and the trajectory will also tend to the equilibrium point. However, as the wind speed gradually increases to make $\widetilde{T}_{m}=13.6$, D-PMSG presents serious chaotic state, forms a chaotic attractor, and enters into an overall unstable chaotic motion. The simulation result is shown in Figure 2(b). $u 3$ is obtained from the input torque Tm provided by the prime mover (WTSs) of the D-PMSG through linear transformation and time-scale transformation, so the change of wind speed will also lead to the system from stable operation to chaos.

3.2. Fractional-Order D-PMSG Wind Turbine Model. Let $\left[x_{1}, x_{2}, x_{3}\right]^{T}=\left[\widetilde{i}_{d}, \widetilde{i}_{q}, \widetilde{w}_{g}\right]^{T}$; the nonlinear D-PMSG model that is considered for analysis is given by the following equation:

$$
\left\{\begin{array}{l}
D_{t}^{\alpha} x_{1}=-x_{1}+x_{2} x_{3}+\widetilde{u}_{d} \\
D_{t}^{\alpha} x_{2}=-x_{2}-x_{1} x_{3}+\mu x_{3}+\widetilde{u}_{d} \\
D_{t}^{\alpha} x_{3}=\sigma\left(x_{2}-x_{3}\right)-\widetilde{T}
\end{array}\right.
$$

wherein the D-PMSG has $\widetilde{T}_{r}=\widetilde{T}_{m}$.

The system parameters are taken as $\sigma=16, \mu=45.92$, $\widetilde{u}_{d}=-0.542, \widetilde{u}_{q}=0.824$, and $\widetilde{T}_{m}=1.16$, and we can calculate three equilibrium points: $\mathrm{E}_{01}=(45.0319,6.7872,6.7147)$, $\mathrm{E}_{02}=(44.8090,-6.6982,-6.7707)$, and $\mathrm{E}_{03}=(-0.5429$, $0.0559,-0.0165)$, respectively. Then, the eigenvalues of the Jacobian matrix at the three equilibrium points are obtained: $(-18.7297,0.3648 \pm 8.7983 \mathrm{i}),(-18.8854,0.4297 \pm 8.78 \mathrm{i})$, and $(-36.7782,-1.0001,19.7782)$. Since each equilibrium point has a positive real part eigenvalue, system (11) will produce an attractor around the equilibrium point and go into the chaotic state.

Taking into account Lemma 7 and Definition 1, we can obtain $\min \left\{\left|\arg \left(\lambda_{i}\right)\right|\right\}=0.9736,0.9689$ for equilibrium points $E_{01}$ and $E_{02}$. When the following inequality (12) is established, the system will show chaotic motion.

$$
\alpha \geq \max _{E_{01}, E_{02}}\left\{\frac{2}{\pi} \min \left[\left|\arg \left(\lambda_{i}\right)\right|\right]\right\}=0.9736 \approx 0.97 .
$$

When $\alpha<0.9689$, system (11) is stable. The initial states are taken as $(20,-5,1)$, and Figures $3(\mathrm{a})$ and $3(\mathrm{~b})$ show the state time-domain diagram and three-phase diagram of the chaotic system of the FOD-PMSG with $\alpha=0.96$ and $\alpha=0.97$, respectively. It can be seen in Figure 3(a) that system (11) is in the quasi-periodic stability state when $\alpha=0.96$. It can be seen from Figure 3(b) that system (11) will have a chaotic attractor and present chaotic motion. Figure 1(c) and Figures 3(a) and 3(b) clearly show that, with the changes of the differential orders, say $\alpha=1, \alpha=0.97$, and $\alpha=0.96$, the state trajectories force the stabilization of the D-PMSG. It is concluded that when $\alpha \in(0.96,1]$, the system will present chaos. From the analysis of chaotic characteristics in this paper, not only the system parameters and external interference will affect the operation characteristics but also the fractional order will affect the motion characteristics. Compared with the integer-order model, the fractionalorder D-PMSG model is more suitable for practical engineering applications.

3.3. T-S Fuzzy State-Space Model. FONS (11) is transformed into the following vector form:

$$
D_{t}^{\alpha} x(t)=A x(t)+\Phi(x(t))+B u(t)
$$

wherein $A=\left[\begin{array}{ccc}-1 & 0 & 0 \\ 0 & -1 & 0 \\ 0 & \sigma & -\sigma\end{array}\right], \Phi(x(t))=\left[\begin{array}{c}x_{2} x_{3} \\ -x_{1} x_{3} \\ 0\end{array}\right]$, and the system external input $u(t)=\left[\widetilde{u}_{d}, \widetilde{u}_{q}, \widetilde{T}_{r}\right]^{T}$.

Considering the external disturbance and parameter uncertainty, the vector form of nonlinear system (11) of the FOD-PMSG is as follows:

$$
\begin{aligned}
D_{t}^{\alpha} x(t) & =\bar{A} x(t)+\bar{B} u(t)+\Phi(x(t))+B_{w} w(t), \\
z(t) & =C x(t)+D_{w} w(t),
\end{aligned}
$$

wherein $\alpha \in(0,1]$ is the fractional order, $x(t)$ is the state vector, $u(t)$ is the control input, $w(t)$ is the disturbance belonging to $L_{2}[0, \infty)$, and $z(t)$ is the performance output. $\bar{A}$ and $\bar{B}$ are the system parameter matrix and input matrix, respectively. $B_{w}, C$, and $D_{w}$ are constant matrixes with proper dimensions.

Consider continuous-time uncertain FONS (14) with T-S fuzzy rules which are described as follows.

Model rule $i$ : if $x_{3}(t)$ is $\mathrm{Mi}$, then

$$
\left\{\begin{array}{l}
D_{t}^{\alpha} x(t)=\bar{A}_{i} x(t)+\bar{B}_{i} u(t)+B_{w i} w(t), \\
z(t)=C_{i} x(t)+D_{w i} w(t), \\
x(0)=x_{0}, \quad i=1,2, \ldots, r,
\end{array}\right.
$$

wherein $r$ is the number of If-Then rules, $M_{i}$ is the fuzzy set, $\bar{B}_{i}$ is an uncertain control input matrix, $\bar{A}_{i}=\left[\begin{array}{ccc}-1 & d_{i} & 0 \\ -d_{i} & -1 & \mu \\ 0 & \sigma & -\sigma\end{array}\right]$, and the value of di is related to $x_{3}(t)$.

Let $\bar{A}_{i}=A_{i}+\Delta A_{i}$ and $\bar{B}_{i}=B_{i}+\Delta B_{i}, A_{i}$ and $B_{i}$ are the known system matrix and input matrix, respectively, while $\Delta A_{i}$ and $\Delta B_{i}$ are real-valued function matrices representing the time-varying parameter uncertainties that have the following form:

$$
\left[\Delta A_{i}, \Delta B_{i}\right]=\left[D_{A i} \Delta F_{A i}(t) E_{A i}, D_{B i} \Delta F_{B i}(t) E_{B i}\right],
$$

wherein $D_{\mathrm{Ai}}, D_{\mathrm{Bi}}, E_{\mathrm{Ai}}$, and $E_{\mathrm{Bi}}$ are known constant matrices and $\Delta F_{\mathrm{Ai}}(t)$ and $\Delta F_{\mathrm{Bi}}(t)$ are unknown matrices with Lebesgue measurable elements satisfying $\Delta F_{A i}^{T}(t) \Delta F_{A i}(t) \leq I$ and $\Delta F_{B i}^{T}(t) \Delta F_{B i}(t) \leq I$.

Through single point fuzzification, fuzzy product reasoning, and center defuzzification, the fuzzy chaos model of the D-PMSG is derived as 


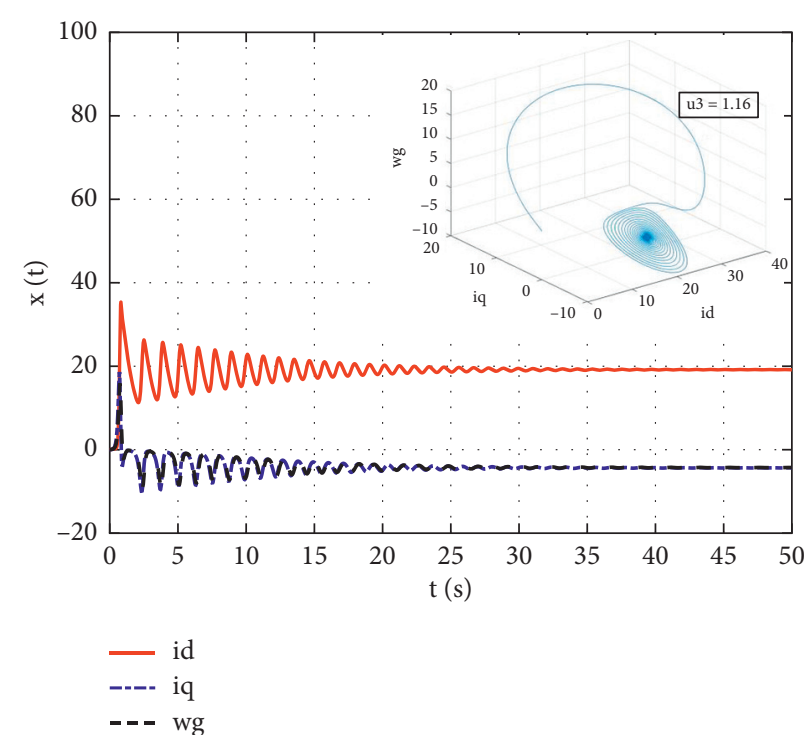

(a)

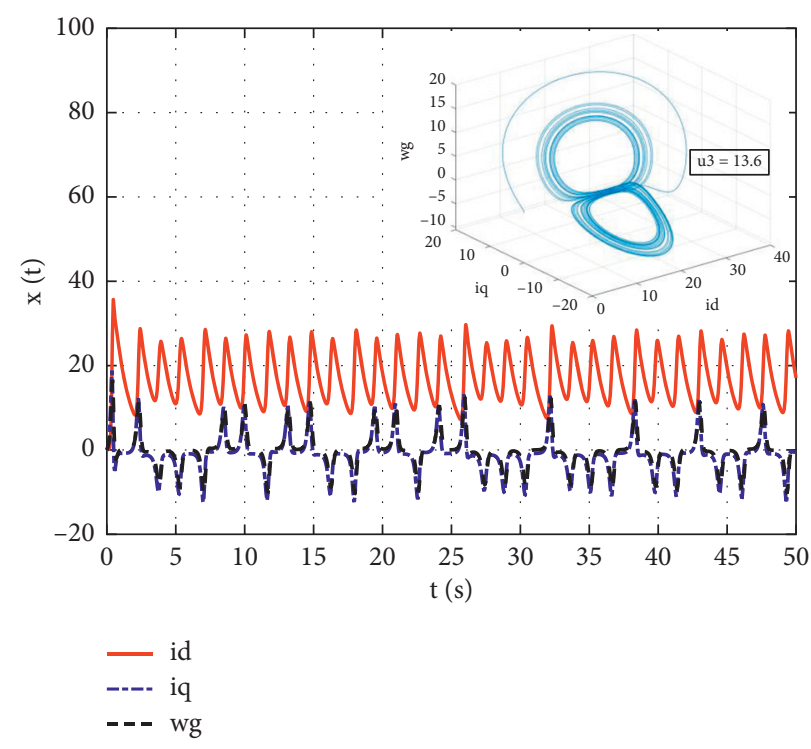

(b)

FIgURE 2: The system chaotic characteristics with $u_{3}$ varying. (a) $u_{3}=1.16$. (b) $u_{3}=13.6$.
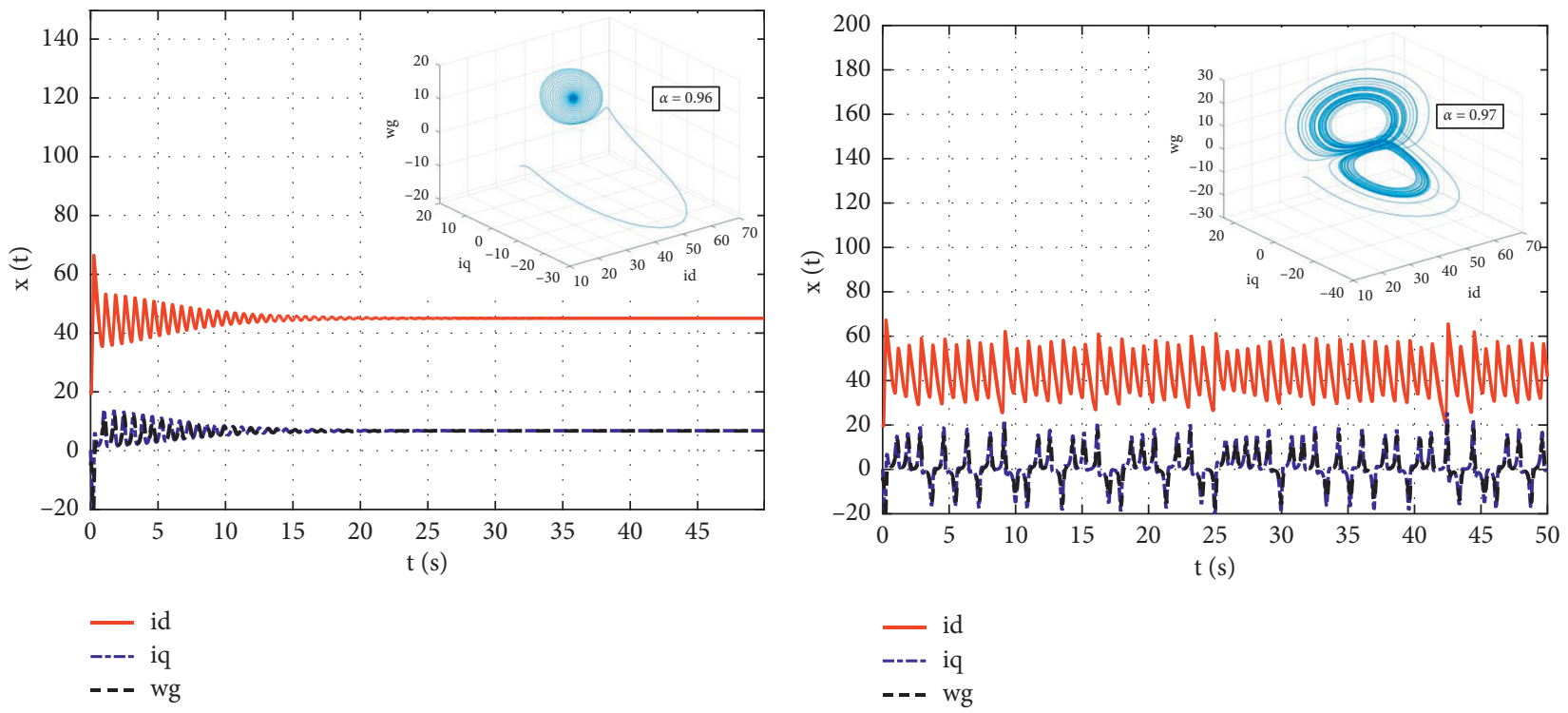

(a)

(b)

FIgURE 3: The system chaotic characteristics with $\alpha$ varying. (a) $\alpha=0.96$. (b) $\alpha=0.97$.

$$
\left\{\begin{array}{l}
D_{t}^{\alpha} x(t)=\sum_{i=1}^{r} h_{i}\left(x_{3}(t)\right)\left[\bar{A}_{i} x(t)+\bar{B}_{i} u(t)+B_{w i} w(t)\right], \\
z(t)=\sum_{i=1}^{r} h_{i}\left(x_{3}(t)\right)\left[C_{i} x(t)+D_{w i} w(t)\right],
\end{array}\right.
$$


wherein $M_{i}\left(x_{3}(t)\right)$ denotes the grade of membership of $x_{3}(t)$ in $M_{\mathrm{i}}$.

$$
\left\{\begin{array}{l}
M_{i}\left(x_{3}(t)\right) \geq 0, \sum_{i=1}^{r} M_{i}\left(x_{3}(t)\right)>0, \\
h_{i}\left(x_{3}(t)\right)=\frac{M_{i}\left(x_{3}(t)\right)}{\sum_{i=1}^{r} M_{i}\left(x_{3}(t)\right)} \\
h_{i}\left(x_{3}(t)\right) \geq 0, \sum_{i=1}^{r} h_{i}\left(x_{3}(t)\right)=1 .
\end{array}\right.
$$

$$
\begin{aligned}
D_{t}^{\alpha} x(t)=\sum_{i=1}^{r} \sum_{j=1}^{r} h_{i} h_{j}\left[\left(\bar{A}_{i}-\bar{B}_{i} K_{j}\right) x(t)+B_{w i} w(t)\right] & =\sum_{i=1}^{r} \sum_{j=1}^{r} h_{i} h_{j}\left[H_{i j} x(t)+B_{w i} w(t)\right], \\
z(t)=\sum_{i=1}^{r} h_{i}\left(x_{3}(t)\right)\left[C_{i} x(t)+D_{w i} w(t)\right], & \\
& \int_{0}^{t_{f}}\left(\gamma^{-1} z^{T}(t) z(t)-\gamma w^{T}(t) w(t)\right) \mathrm{d} t \leq 0 .
\end{aligned}
$$

$\forall t_{f}>0$ and $w(t), \in L_{2}[0, \infty), \gamma$ is the system external disturbance suppression.

Theorem 1. System (21) is globally Mittag-Leffler stable if there exist a symmetric positive definite matrix $P$, matrixes $M_{i}$, and positive constants $\gamma, \varepsilon_{i 1}, \varepsilon_{i 2}, \eta_{i}, \eta_{j}, \delta_{i}$, and $\delta_{j}(i=1$, $2, \ldots, r ; 1 \leq i<j \leq r)$ such that the following LMIs hold:

$$
\left[\begin{array}{cccccc}
\prod_{i i 11} & B_{w i} & \mathrm{QE}_{A i}^{T} & M_{i}^{T} E_{B i}^{T} & \mathrm{QC}_{i}^{T} & 0 \\
* & -\gamma I & 0 & 0 & 0 & D_{w i}^{T} \\
* & * & -\varepsilon_{i 1} I & 0 & 0 & 0 \\
* & * & * & -\varepsilon_{i 2} I & 0 & 0 \\
* & * & * & * & -0.5 \gamma I & 0 \\
* & * & * & * & * & -0.5 \gamma I
\end{array}\right]<0, \quad i=1,2, \ldots, r,
$$




$$
\left[\begin{array}{cccccccc}
\Pi_{i j 11} & B_{w i j} & \mathrm{QE}_{A i}^{T} & M_{j}^{T} E_{B i}^{T} & \mathrm{QE}_{A j}^{T} & M_{i}^{T} E_{B j}^{T} & \mathrm{QC}_{i j}^{T} & 0 \\
* & -\gamma I & 0 & 0 & 0 & 0 & 0 & D_{w i j}^{T} \\
* & * & -\eta_{i} I & 0 & 0 & 0 & 0 & 0 \\
* & * & * & -\delta_{i} I & 0 & 0 & 0 & 0 \\
* & * & * & * & -\eta_{j} I & 0 & 0 & 0 \\
* & * & * & * & * & -\delta_{j} I & 0 & 0 \\
* & * & * & * & * & * & -0.5 \gamma I & 0 \\
* & * & * & * & * & * & * & -0.5 \gamma I
\end{array}\right]<0, \quad 1 \leq i<j \leq r,
$$

wherein $\Pi_{i i 11}=\operatorname{Sym}\left(A_{i} Q-B_{i} M_{i}\right)+\varepsilon_{i 1} D_{A i} D_{A i}^{T}+\varepsilon_{i 2} D_{B i} D_{B i}^{T}$,

$$
\Pi_{i j 11}=\operatorname{Sym}\left(A_{i} Q-B_{i} M_{j}+A_{j} Q-B_{j} M_{i}\right)+\eta_{i} D_{A i} D_{A i}^{T}+\delta_{i} D_{B i} D_{B i}^{T}+\eta_{j} D_{A j} D_{A j}^{T}+\delta_{j} D_{B j} D_{B j}^{T} .
$$

Remark 2. $\operatorname{Sym}\{X\}$ is used to denote the expression $X T+X$ and Sym.

Proof. For fuzzy system (21), we consider the following Lyapunov functional candidate $V(x(t))=x^{T}(t) \mathrm{Px}(t)$, where $\mathrm{P}$ is a feasible solution of conditions (23) and (24). From Lemma 1 , it is easy to verify that

$$
\lambda_{\min }(P)\|x(t)\|^{2} \leq V(x(t)) \leq \lambda_{\max }(P)\|x(t)\|^{2} .
$$

Therefore, inequality (5) in Lemma 1 is satisfied. When $w(t) \equiv 0$ and $z(t) \equiv 0$, it can be seen from Lemma 2 and equation (21) that we obtain the Caputo derivative of $V(x(t))$ as follows:

$$
\begin{aligned}
D_{t}^{\alpha} V(x(t)) \leq 2 x^{T}(t) P D_{t}^{\alpha} x(t) & =\sum_{i=1}^{r} \sum_{j=1}^{r} h_{i} h_{j} 2 x^{T}(t) \mathrm{PH}_{i j} x(t) \\
& =\sum_{i=1}^{r} \sum_{j=1}^{r} h_{i} h_{j} 2 x^{T}(t) P\left[\left(A_{i}-B_{i} K_{j}\right)+D_{A i} \Delta F_{A i}(t) E_{A i}-D_{B i} \Delta F_{B i}(t) E_{B i} K_{j}\right] x(t) .
\end{aligned}
$$

By applying Lemma 3 to (27), one obtains that (27) holds if and only if there exist real constants $\zeta_{i 1}>0$ and $\zeta_{i 2}>0$ $(i=1,2, \ldots, r)$ such that

$$
\begin{aligned}
D_{t}^{\alpha} & V(x(t)) \leq \sum_{i=1}^{r} \sum_{j=1}^{r} h_{i} h_{j} x^{T}(t)\left\{\operatorname{Sym}\left[P\left(A_{i}-B_{i} K_{j}\right)\right]+2 \mathrm{PD}_{A i} \Delta F_{A i}(t) E_{A i}-2 \mathrm{PD}_{B i} \Delta F_{B i}(t) E_{B i} K_{j}\right\} x(t) \\
= & \sum_{i=1}^{r} \sum_{j=1}^{r} h_{i} h_{j}\left\{x^{T}(t) \operatorname{Sym}\left[P\left(A_{i}-B_{i} K_{j}\right)\right] x(t)+2 x^{T}(t) \mathrm{PD}_{A i} \Delta F_{A i}(t) E_{A i} x(t)-2 x^{T}(t) \mathrm{PD}_{B i} \Delta F_{B i}(t) E_{B i} K_{j} x(t)\right\} \\
= & \sum_{i=1}^{r} \sum_{j=1}^{r} h_{i} h_{j}\left\{x^{T}(t) \operatorname{Sym}\left[P\left(A_{i}-B_{i} K_{j}\right)\right] x(t)+\zeta_{i 1} x^{T}(t) \mathrm{PD}_{A i} D_{A i}^{T} P x(t)+\zeta_{i 1}^{-1} x^{T}(t) E_{A i}^{T} E_{A i} x(t)+\zeta_{i 2} x^{T}(t)\right. \\
& \left.\mathrm{PD}_{B i} D_{B i}^{T} P x(t)+\zeta_{i 2}^{-1} x^{T}(t) K_{j}^{T} E_{B i}^{T} E_{B i} K_{j} x(t)\right\} \\
= & \sum_{i=1}^{r} \sum_{j=1}^{r} h_{i} h_{j} x^{T}(t) \Omega_{i} x(t),
\end{aligned}
$$


wherein $\quad \Omega_{i}=\operatorname{Sym}\left[P\left(A_{i}-B_{i} K_{j}\right)\right]+\zeta_{i 1} \mathrm{PD}_{A i} D_{A i}^{T} P+\zeta_{i 1}^{-1} E_{A i}^{T}$ $E_{A i}+\zeta_{i 2} \mathrm{PD}_{B i} D_{B i}^{T} P+\zeta_{i 2}^{-1} K_{j}^{T} E_{B i}^{T} E_{B i} K_{j}$.

From equation (28), it is easy to conclude that the following inequality holds:

$$
D_{t}^{\alpha} x(t) \leq \sum_{i=1}^{r} \sum_{j=1}^{r} h_{i} h_{j}\left[\lambda_{\max }\left(\Omega_{i}\right)\|x(t)\|^{2}\right] .
$$

Thus, condition (2) in Lemma 1 is also satisfied. It can be concluded that system (21) is Mittag-Leffler stable under $w(t) \equiv 0$ and $z(t) \equiv 0$.

To show system (21) is Mittag-Leffler stable with Ho robust control performance index $\gamma$, according to Lemma 5 , we have the following estimate:

$$
\begin{aligned}
D_{t}^{\alpha} V & (x(t))+\gamma^{-1} z^{T}(t) z(t)-\gamma w^{T}(t) w(t) \\
\leq & \sum_{i=1}^{r} \sum_{j=1}^{r} h_{i} h_{j}\left[x^{T}(t)\left(\mathrm{PH}_{i j}+H_{i j}^{T} P\right) x(t)+2 \gamma^{-1} x^{T}(t) C_{i}^{T} C_{i} x(t)+2 x^{T}(t) \mathrm{PB}_{w i} w(t)+2 \gamma^{-1} w^{T}(t) D_{w i}^{T} D_{w i} w(t)-\gamma w^{T}(t) w(t)\right] \\
= & \sum_{i=1}^{r} h_{i}^{2}\left[x^{T}(t) \operatorname{Sym}\left(\mathrm{PH}_{i i}\right) x(t)+2 \gamma^{-1} x^{T}(t) C_{i}^{T} C_{i} x(t)+2 x^{T}(t) \mathrm{PB}_{w i} w(t)+2 \gamma^{-1} w^{T}(t) D_{w i}^{T} D_{w i} w(t)-\gamma w^{T}(t) w(t)\right] \\
& +\sum_{i=1}^{r} \sum_{i<j} h_{i} h_{j}\left[x^{T}(t) \operatorname{Sym}\left(P\left(H_{i j}+H_{j i}\right)\right) x(t)+2 \gamma^{-1} x^{T}(t) C_{i j}^{T} C_{i j} x(t)+2 x^{T}(t) \mathrm{PB}_{w i j} w(t)+2 \gamma^{-1} w^{T}(t) D_{w i j}^{T} D_{w i j} w(t)-\gamma w^{T}(t) w(t)\right],
\end{aligned}
$$

wherein $C_{\mathrm{ij}}=C_{i}+C_{j}, B_{\mathrm{wij}}=B_{\mathrm{wi}}+B_{\mathrm{wj}}$, and $D_{\mathrm{wij}}=D_{\mathrm{wi}}+D_{\mathrm{wj}}$.

If it is possible to assume that each sum of the second equation in (30) is negative definite, respectively, then system (21) is Mittag-Leffler stable.
Firstly, assume that the first sum of the last equation in (30) is negative definite.

$$
\sum_{i=1}^{r} h_{i}^{2}\left[x^{T}(t) \operatorname{Sym}\left(\mathrm{PH}_{i i}\right) x(t)+2 \gamma^{-1} x^{T}(t) C_{i}^{T} C_{i} x(t)+2 x^{T}(t) \mathrm{PB}_{w i} w(t)+2 \gamma^{-1} w^{T}(t) D_{w i}^{T} D_{w i} w(t)-\gamma w^{T}(t) w(t)\right]<0 .
$$

In view of the membership function being less than 1 , by Lemma 3 , there exist positive scalars $\varepsilon_{i 1}$ and $\varepsilon_{i 2}(i=1,2, \ldots, \mathrm{r})$ such that

$$
\begin{gathered}
\sum_{i=1}^{r} h_{i}^{2}\left\{x^{T}(t)\left[\operatorname{Sym}\left(P\left(A_{i}-B_{i} K_{i}\right)\right)+\varepsilon_{i 1} \mathrm{PD}_{A i} D_{A i}^{T} P+\varepsilon_{i 1}^{-1} E_{A i}^{T} E_{A i}+\varepsilon_{i 2} \mathrm{PD}_{B i} D_{B i}^{T} P+\varepsilon_{i 2}^{-1} K_{i}^{T} E_{B i}^{T} E_{B i} K_{i}+2 \gamma^{-1} C_{i}^{T} C_{i}\right]\right. \\
\left.x(t)+2 x^{T}(t) \mathrm{PB}_{w i} w(t)+2 \gamma^{-1} w^{T}(t) D_{w i}^{T} D_{w i} w(t)-\gamma w^{T}(t) w(t)\right\}<0 .
\end{gathered}
$$

Inequation (32) can be represented by

$$
\sum_{i=1}^{r} h_{i}^{2}\left[x^{T}(t) w^{T}(t)\right]\left[\begin{array}{cc}
\Phi_{i i 11} & \Phi_{i i 12} \\
* & \Phi_{i i 22}
\end{array}\right]\left[\begin{array}{c}
x(t) \\
w(t)
\end{array}\right]<0,
$$

wherein

$$
\begin{aligned}
& \Phi_{i i 11}=\text { Sym } \\
& \left.\left(A_{i}-B_{i} K_{i}\right)\right]+\varepsilon_{i 1} \mathrm{PD}_{A i} D_{A i}{ }^{T} P+\varepsilon_{i 1}{ }^{-1} E_{A i}{ }^{T} \mathrm{E}_{A i}{ }^{+} \\
& \varepsilon_{i 2} \mathrm{PD}_{B i} D_{B i}{ }^{T} P+\varepsilon_{i 2}{ }^{-1} K_{i}^{T} E_{B i}{ }^{T} E_{B i} K_{i}+2 \gamma^{-1} C_{i}^{\mathrm{T}} C_{i}, \\
& \Phi_{i i 12}=\mathrm{PB}_{w i}, \\
& \Phi_{i i 22}=2 \gamma^{-1} D_{w i}{ }^{\mathrm{T}} D_{w i}+\gamma I .
\end{aligned}
$$

Then,

$$
\Phi=\left[\begin{array}{cc}
\Phi_{i i 11} & \Phi_{i i 12} \\
* & \Phi_{i i 22}
\end{array}\right]<0, \quad i=1,2, \ldots, r .
$$

Now, pre- and postmultiply both sides of inequality (34) with the transformation matrix diag $[P-1, I]$, and let $\mathrm{Q}=\mathrm{P}-1$ and $M_{i}=K_{i P}{ }^{-1}$; we see that $\Phi<0$ is equivalent to the following condition:

$$
\left[\begin{array}{cc}
\Xi_{i i 11} & \Xi_{i i 12} \\
* & \Xi_{i i 22}
\end{array}\right]<0, \quad i=1,2, \ldots, r
$$

wherein 
$\Xi_{i i 11}=\operatorname{Sym}\left(A_{i} Q-B_{i} M_{i}\right)$

$+\varepsilon_{i 1} D_{A i} D_{A i}{ }^{\mathrm{T}}+\varepsilon_{i 1}{ }^{-1} \mathrm{QE}_{A i}{ }^{\mathrm{T}} E_{A i} Q+\varepsilon i_{2} D_{B i} D_{B i}{ }^{\mathrm{T}}+$

$\varepsilon_{i 2}{ }^{-1} M_{i}^{\mathrm{T}} E_{B i}{ }^{\mathrm{T}} E_{B i} M_{i}+2 \gamma^{-1} \mathrm{QC}_{i}^{\mathrm{T}} C_{i} \mathrm{Q}$,

$\Xi_{i i 12}=B_{w i}$,

$\Xi_{i i 22}=2 \gamma^{-1} D_{w i}{ }^{\mathrm{T}} D_{w i}+\gamma$.
By Lemma 4 (Schur complement), we have the first LMI (23) in Theorem 1.

Secondly, LMI (24) can be established through a similar procedure. Assume that the second sum of the last equation in (30) is negative definite.

$$
\sum_{i=1}^{r} \sum_{i<j} h_{i} h_{j}\left[x^{T}(t) \operatorname{Sym}\left(P\left(H_{i j}+H_{j i}\right)\right) x(t)+2 \gamma^{-1} x^{T}(t) C_{i j}^{T} C_{i j} x(t)+2 x^{T}(t) \mathrm{PB}_{w i j} w(t)+2 \gamma^{-1} w^{T}(t) D_{w i j}^{T} D_{w i j} w(t)-\gamma w^{T}(t) w(t)\right]<0 .
$$

In view of the membership function being less than 1, by applying Lemma 3 to (36), one obtains that (36) holds if and only if there exist positive scalars $\eta_{i}, \delta_{i}, \eta_{j}$, and $\delta_{j}(i=1$, $2, \ldots, r, i<j)$ such that

$$
\begin{aligned}
& \sum_{i=1}^{r} \sum_{i<j} h_{i} h_{j}\left\{x ^ { T } ( t ) \left[\operatorname{Sym}\left(P\left(A_{i}-B_{i} K_{j}+A_{j}-B_{j} K_{i}\right)\right)+\eta_{i} \mathrm{PD}_{A i} D_{A i}^{T} P+\eta_{i}^{-1} E_{A i}^{T} E_{A i}+\delta_{i} \mathrm{PD}_{B i} D_{B i}^{T} P\right.\right. \\
& \left.\quad+\delta_{i}^{-1} K_{j}^{T} E_{B i}^{T} E_{B i} K_{j}+\eta_{j} \mathrm{PD}_{A j} D_{A j}^{T} P+\eta_{j}^{-1} E_{A j}^{T} E_{A j}+\delta_{j} \mathrm{PD}_{B j} D_{B j}^{T} P+\delta_{j}^{-1} K_{i}^{T} E_{B j}^{T} E_{B j} K_{i}+2 \gamma^{-1} C_{i j}^{T} C_{i j}\right] \\
& \left.x(t)+2 x^{T}(t) \mathrm{PB}_{w i j} w(t)+2 \gamma^{-1} w^{T}(t) D_{w i j}^{T} D_{w i j} w(t)-\gamma w^{T}(t) w(t)\right\}<0 .
\end{aligned}
$$

Then,

$$
\sum_{i=1}^{r} \sum_{i<j} h_{i} h_{j}\left[x^{T}(t) w^{T}(t)\right]\left[\begin{array}{cc}
\Phi_{i j 11} & \Phi_{i j 12} \\
* & \Phi_{i j 22}
\end{array}\right]\left[\begin{array}{c}
x(t) \\
w(t)
\end{array}\right]<0,
$$

wherein

$$
\begin{aligned}
& \Phi_{i j 11}=\operatorname{Sym}_{\mathrm{T}}\left[P\left(A_{i}-B_{i} K_{j}+A_{j}-B_{j} K_{i}\right)\right]+\eta_{i} \mathrm{PD}_{A i} D_{A i}{ }^{\mathrm{T}} \\
& P+\eta_{i}{ }^{-1} E_{A i} E_{A i}+ \\
& \eta_{i} \mathrm{PD}_{B i} D_{B i}{ }^{\mathrm{T}} P+\eta_{i}{ }^{-1} K_{j}^{\mathrm{T}} E_{B i}{ }^{\mathrm{T}} E_{B i} K_{j}+\eta_{j} \mathrm{PD}_{A j} D_{A j}{ }^{\mathrm{T}} P+\eta_{j}{ }^{-1} \\
& E_{A j}{ }^{\mathrm{T}} E_{A j}+\delta_{j} \mathrm{PD}_{B j} D_{B j}{ }^{\mathrm{T}} P+\delta_{j}{ }^{-1} K_{i}^{\mathrm{T}} E_{B j} E_{B j} K_{i}+2 \gamma^{-1} C_{i j}{ }^{\mathrm{T}} C_{i j}, \\
& \Phi_{i j 12}=\mathrm{PB}_{w i j}, \\
& \Phi_{i j 22}=2 \gamma^{-1} D_{w i j}{ }^{\mathrm{T}} D_{w i j}+\gamma I .
\end{aligned}
$$

Inequation (38) can be represented by

$$
\widetilde{\Phi}=\left[\begin{array}{cc}
\Phi_{i j 11} & \Phi_{i j 12} \\
* & \Phi_{i j 22}
\end{array}\right]<0, \quad 1 \leq i<j \leq r .
$$

Pre- and postmultiply both sides of (39) with the transformation matrix $\operatorname{diag}[P-1, I]$, and let $Q=P-1$ and $M_{i}=K_{i P}-1$; we see that $\widetilde{\Phi}<0$ is equivalent to the following condition:

$$
\left[\begin{array}{cc}
\Xi_{i j 11} & \Xi_{i j 12} \\
* & \Xi_{i j 22}
\end{array}\right]<0, \quad 1 \leq i<j \leq r,
$$

wherein

$$
\begin{aligned}
& \Xi_{i j 11}=\operatorname{Sym}\left(A_{i} Q-B_{i} M_{j}+A_{j} Q-B_{j} M_{i}\right)+\eta_{i} D_{A i} D_{A i}{ }^{\mathrm{T}}+ \\
& \eta_{i}{ }^{-1} \mathrm{QE}_{A i}{ }^{\mathrm{T}} E_{A i} Q+\delta_{i} D_{B i} D_{B i}{ }^{\mathrm{T}}+\delta_{i}{ }^{-1} M_{j}{ }^{\mathrm{T}} E_{B i}{ }^{\mathrm{T}} E_{B i} M_{j}{ }^{\mathrm{T}} \\
& \eta_{j} D_{A j} D_{A j}{ }^{\mathrm{T}}+\eta_{j}{ }^{-1} \mathrm{QE}_{A j}{ }^{\mathrm{T}} E_{A j} Q+\delta_{j} D_{B j} D_{B j}{ }^{\mathrm{T}}+\delta_{j}{ }^{-1} M_{i}^{\mathrm{T}}{ }^{\mathrm{T}}{ }_{B j}{ }^{\mathrm{T}} E \\
& { }_{B j} M_{i}+2 \gamma^{-1} \mathrm{Q} C_{i j}{ }^{\mathrm{T}} C_{i j} Q, \\
& \Xi_{i j 12}=B_{w i j} \\
& \Xi_{i j 22}=2 \gamma^{-1} D_{w i j}{ }^{\mathrm{T}} D_{w i j}+\gamma I .
\end{aligned}
$$

By applying Lemma 4, equation (40) is equivalent to the second LMI (24) in Theorem 1.

When both (23) and (24) are established, we can obtain

$$
D_{t}^{\alpha} V(x(t))+\gamma^{-1} z^{T}(t) z(t)-\gamma w^{T}(t) w(t) \leq 0 .
$$
have

Integrating (41) with respect to $t$ from 0 to $t_{f}$ one can

$$
I_{t_{f}}^{1} D_{t_{f}}^{\alpha} V(x(t))+\int_{0}^{t_{f}} \gamma^{-1} z^{T}(t) z(t)-\gamma w^{T}(t) w(t) \mathrm{d} t \leq 0 .
$$

By using Property 1 and Lemma 6, we have

$$
\begin{aligned}
I_{t_{f}}^{1} D_{t_{f}}^{\alpha} V(x(t)) & =I_{t_{f}}^{1-\alpha} I_{t_{f}}^{\alpha} D_{t_{f}}^{\alpha} V(x(t))=I_{t_{f}}^{1-\alpha}\left(I_{t_{f}}^{\alpha} D_{t_{f}}^{\alpha} V(x(t))\right) \\
& =I_{t_{f}}^{1-\alpha}(V(x(t))-V(x(0)))=I_{t_{f}}^{1-\alpha} V(x(t))-I_{t_{f}}^{1-\alpha} V(x(0)) .
\end{aligned}
$$


On the contrary, according to Definition 2, we can have $I_{t_{f}}^{1-\alpha} V(x(t))=\frac{1}{\Gamma(1-\alpha)} \int_{0}^{t_{f}}\left(t_{f}-\tau\right)^{-\alpha} x^{T}(\tau) P x(\tau) \mathrm{d} \tau$,

wherein $\forall \quad t_{f}>\tau, \quad \alpha \in(0,1], \quad$ and $\quad\left(t_{f}-\tau\right)-\alpha>0$, $I_{t_{f}}^{1-\alpha} V(x(t))>0$.

Under the zero initial condition, we obtain

$$
\int_{0}^{t_{f}} \gamma^{-1} z^{T}(t) z(t)-\gamma w^{T}(t) w(t) \mathrm{d} t<0
$$

By Definition 3, system (21) is globally Mittag-Leffler stable with $\mathrm{H} \infty$ performance index $\gamma$. This completes the proof.

\section{Numerical Simulations}

In this section, the dynamic characteristics of the FODPMSG are explored, and their corresponding effect is reflected in model (11). Suppose $x_{3}(t) \in[-30,30]$, the representation of nonlinear model (11) with T-S fuzzy rules is described as follows:

$$
\begin{aligned}
& \mathrm{R}^{1}: \text { if } x_{3}(t) \text { is } M_{1} \text {, then } \\
& \begin{aligned}
D_{t}^{\alpha} x(t) & =\bar{A}_{1} x(t)+\bar{B}_{1} u(t)+B_{w 1} w(t), \\
z(t) & =C_{1} x(t)+D_{w 1} w(t)
\end{aligned}
\end{aligned}
$$

$\mathrm{R}^{2}:$ if $x_{3}(t)$ is $M_{2}$, then

$$
\begin{aligned}
D_{t}^{\alpha} x(t) & =\bar{A}_{2} x(t)+\bar{B}_{2} u(t)+B_{w 2} w(t), \\
z(t) & =C_{2} x(t)+D_{w 2} w(t),
\end{aligned}
$$

wherein $M_{1,2}$ are fuzzy sets, $\bar{A}_{1,2}$ are uncertain system parameter matrixes, $\bar{B}_{1,2}$ are uncertain control input matrixes, $B_{w 1,2}$ are matrixes for external disturbance, and $C_{1,2}$ and $A_{w 1,2}$ are performance output matrixes with appropriate dimensions.

When $u(t)=0$, we have the following open-loop fuzzy FOD-PMSG with parameter uncertainty and external disturbance:

$$
\begin{array}{r}
D_{t}^{\alpha} x(t)=\sum_{i=1}^{2} h_{i}\left(x_{3}(t)\right)\left[\left(A_{i}+\Delta A_{i}\right) x(t)+B_{w i} w(t)\right] \\
z(t)=\sum_{i=1}^{2} h_{i}\left(x_{3}(t)\right)\left[C_{i} x(t)+D_{w i} w(t)\right] .
\end{array}
$$

Take the system uncertainty parameters as $\sigma=16 \pm 0.2$ and $\mu=45.92 \pm 1, \mu$ is random in the interval [44.92, 26.92], and we have $\Delta \sigma=0.2, \Delta \mu=\sin (t)$,

$$
\begin{aligned}
& A_{1}=\left[\begin{array}{ccc}
-1 & 30 & 0 \\
-30 & -1 & 45.92 \\
0 & 16 & -16
\end{array}\right], A_{2}=\left[\begin{array}{ccc}
-1 & -30 & 0 \\
30 & -1 & 45.92 \\
0 & 16 & -16
\end{array}\right], \Delta A_{1}=\Delta A_{2}=\left[\begin{array}{ccc}
0 & 0 & 0 \\
0 & 0 & \sin (t) \\
0 & 0.2 & -0.2
\end{array}\right], \\
& D_{A i} \Delta F_{A i}(t) E_{A i}=\left[\begin{array}{ccc}
0 & 0 & 0 \\
0 & 0.0625 & 0 \\
0 & 0.2 & 0.2296
\end{array}\right]\left[\begin{array}{ccc}
1 & 0 & 0 \\
0 & \sin (t) & 0 \\
0 & 0 & 1
\end{array}\right]\left[\begin{array}{ccc}
0 & 0 & 0 \\
0 & 0 & 16 \\
0 & 0.871 & -0.871
\end{array}\right] \text {, } \\
& D_{A 1}=D_{A 2}=\left[\begin{array}{ccc}
0 & 0 & 0 \\
0 & 0.0625 & 0 \\
0 & 0.2 & 0.2296
\end{array}\right], E_{A 1}=E_{A 2}=\left[\begin{array}{ccc}
0 & 0 & 0 \\
0 & 0 & 16 \\
0 & 0.871 & -0.871
\end{array}\right] \text {. }
\end{aligned}
$$

For demonstrating the robustness and applicability of the proposed control scheme, the disturbance matrix and external disturbance in the simulation are assumed to be $B_{w 1}=B_{w 2}=\left[\begin{array}{c}0 \\ 0 \\ 0.5\end{array}\right]$ and $w(t)=\sin (2 \pi t) e^{-0.5 t}$.

We consider output parameters $C_{1}=C_{2}=[10,10,20]$ and $D_{w 1}=D_{w 2}=1$ and the membership functions

$$
h_{1}\left(x_{3}(t)\right)=\frac{1}{2}\left(1+\frac{x_{3}(t)}{30}\right), h_{2}\left(x_{3}(t)\right)=\frac{1}{2}\left(1-\frac{x_{3}(t)}{30}\right) \text {. }
$$

When $u(t)=0$, the system state diagrams and threephase diagrams of the FOD-PMSG with different differential orders are shown in Figure 4. Figure 4(a) depicts the chaotic behavior of solutions plotted for integer order $\alpha=1$. Figure 4(b) shows some variations in solutions when the order is chosen as $\alpha=0.97$. Further changes in order, say $\alpha=0.96$, will affect the behavior of the states which are picturized in Figure 4(c). From Figure 4(d), it is clear that changes in the differential order will drastically affect the solutions to have stable states for order $\alpha=0.95$.

Figure 4 clearly shows the nature of solutions for various differential orders. In addition, it can be observed that, with the changes of the differential orders, say $\alpha=1,0.97,0.96$, and 0.95 , the state trajectories force the stabilization of the D-PMSG, which is intuitively reasonable.

By using PDC technology, the fuzzy controller can be designed as follows: 

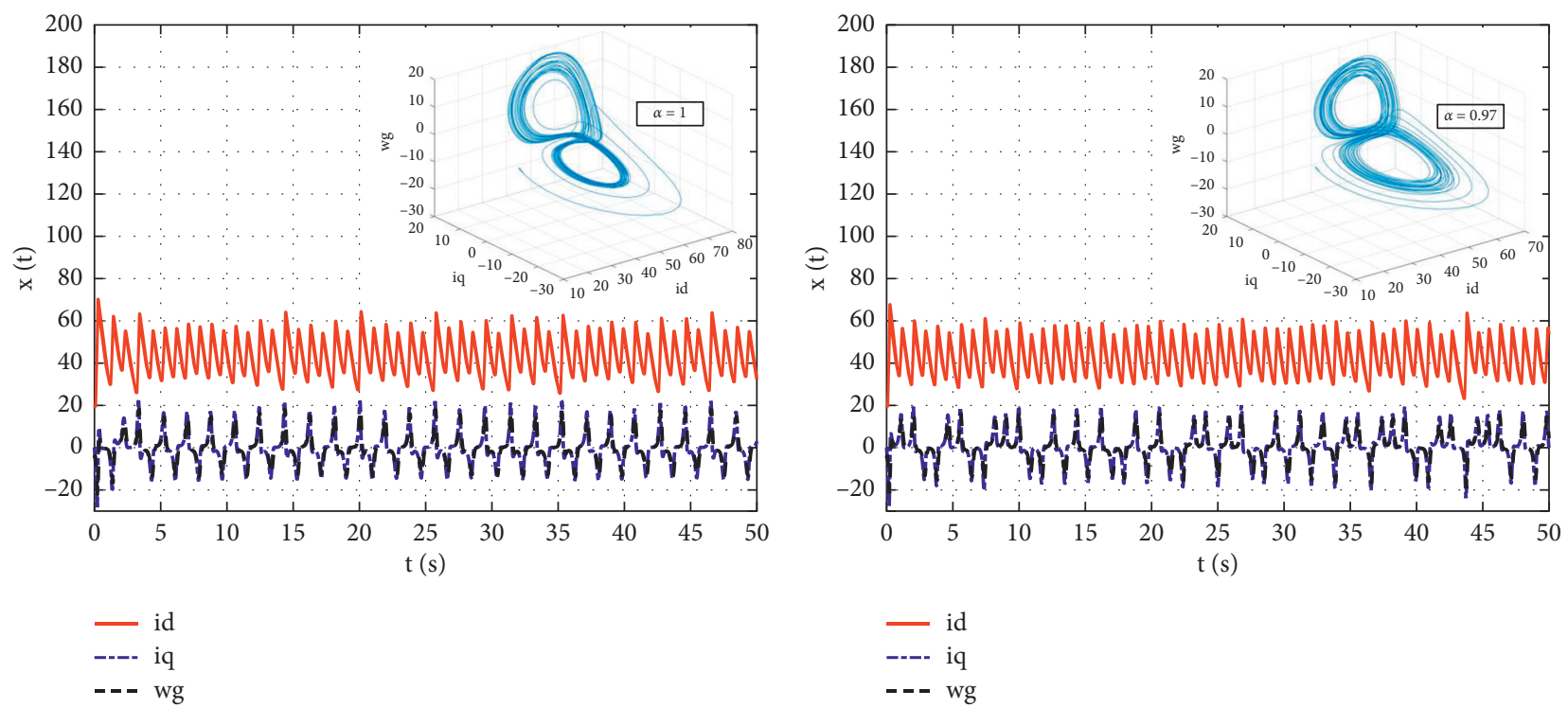

(a)

(b)
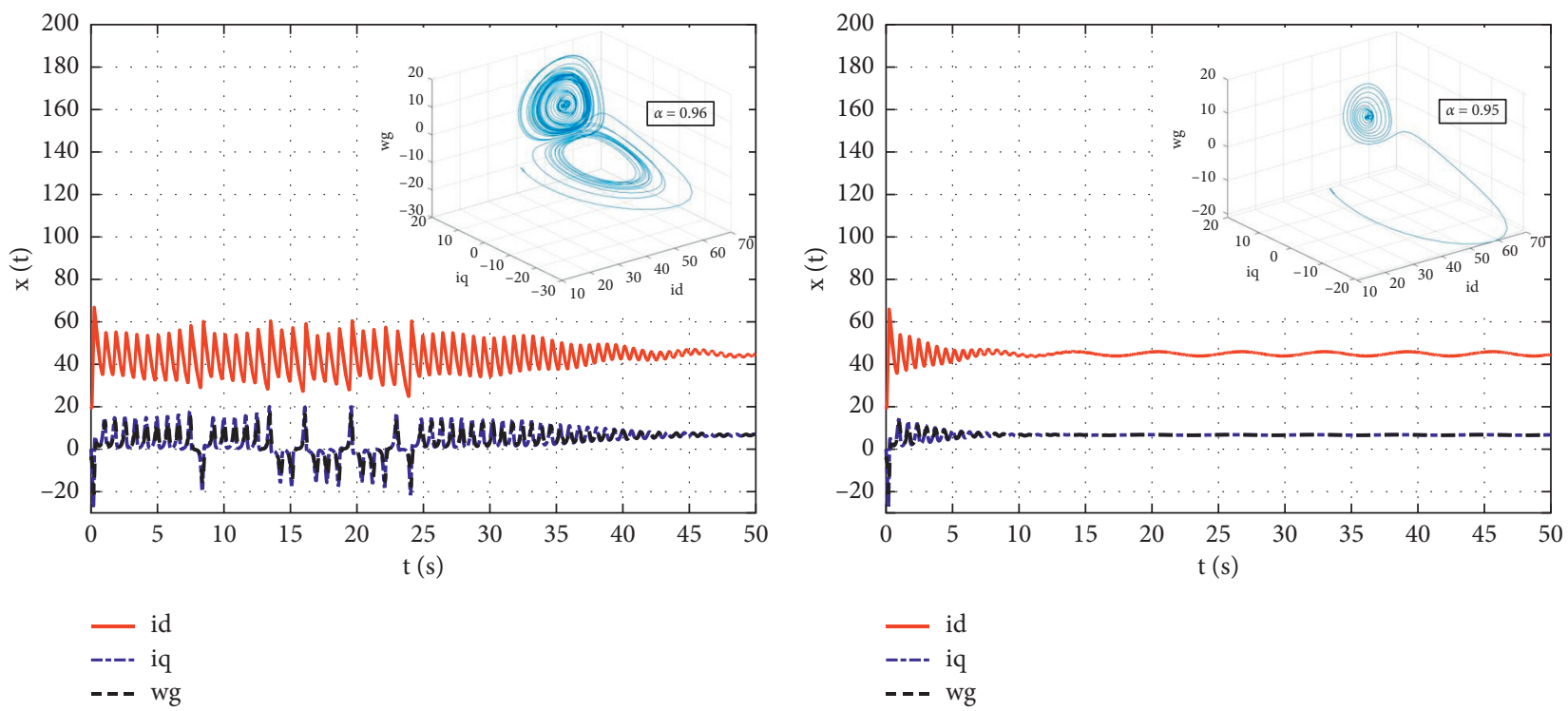

(c)

(d)

FIGURE 4: System state diagrams and three-phase diagrams without control. (a) $\alpha=1$. (b) $\alpha=0.97$. (c) $\alpha=0.96$. (d) $\alpha=0.95$.

$\mathrm{R}^{1}:$ if $x_{3}(t)$ is $M_{1}$, then $u(t)=-K_{1} x(t)$

$\mathrm{R}^{2}$ : if $x_{3}(t)$ is $M_{2}$, then $u(t)=-K_{2} x(t)$

Then, the fuzzy state feedback controller can be obtained:

$$
u(t)=-\sum_{j=1}^{2} h_{j}\left(x_{3}(t)\right) K_{j} x(t) .
$$

Taking system parameter uncertainties and external disturbance into account, FOD-PMSG can be modeled as the following uncertain continuous-time fuzzy closed-loop models:

$$
\begin{aligned}
D_{t}^{\alpha} x(t) & =-\sum_{i=1}^{2} \sum_{j=1}^{2} h_{i} h_{j}\left[\left(A_{i}-B_{i} K_{j}+\Delta A_{i}-\Delta B_{i} K_{j}\right) x(t)+B_{w i} w(t)\right] \\
z(t) & =\sum_{i=1}^{2} h_{i}\left[C_{i} x(t)+D_{w i} w(t)\right]
\end{aligned}
$$




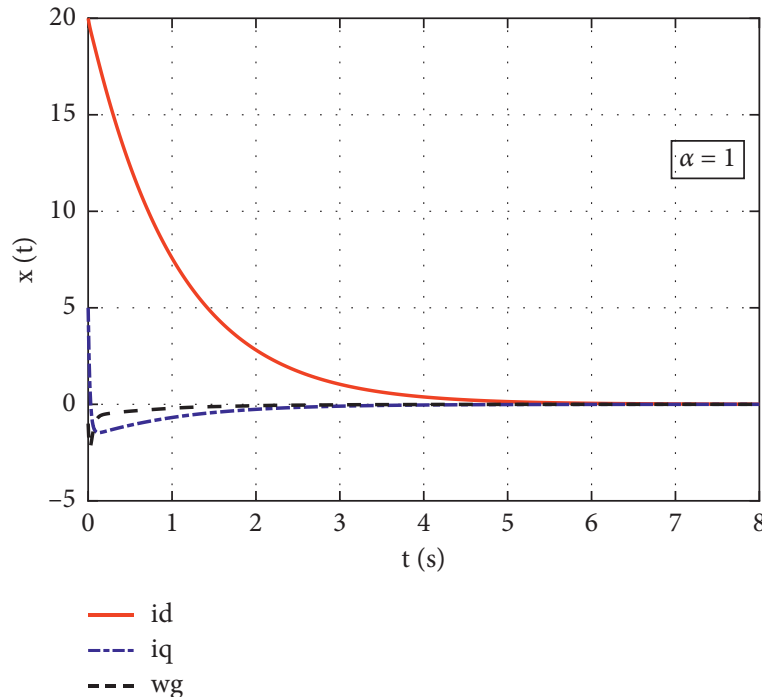

(a)

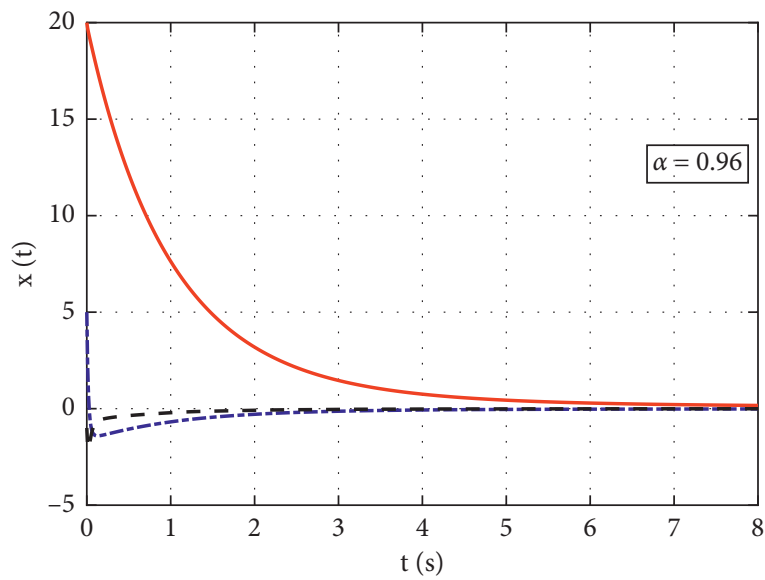

$\begin{array}{ll}\text { - } & \text { id } \\ --- & \text { iq } \\ --- & \text { wg }\end{array}$

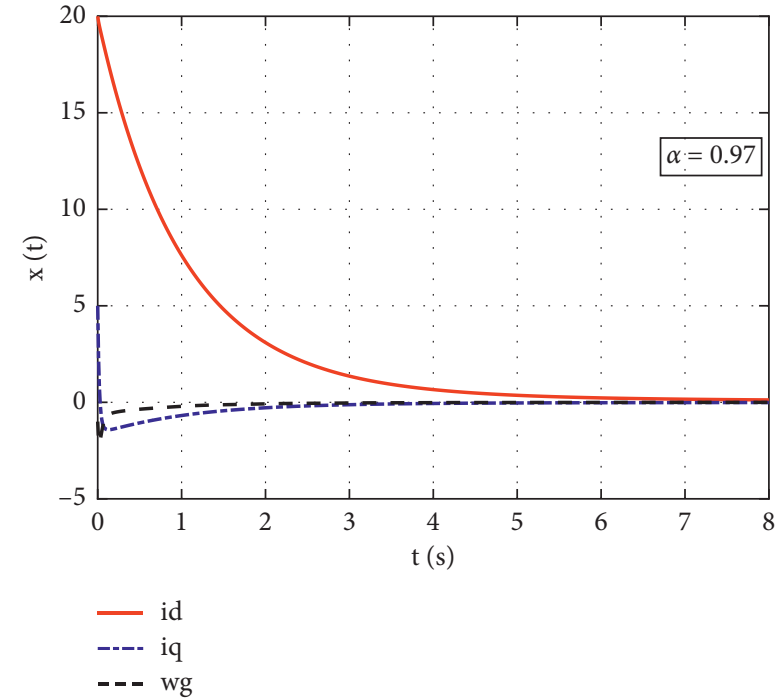

(b)

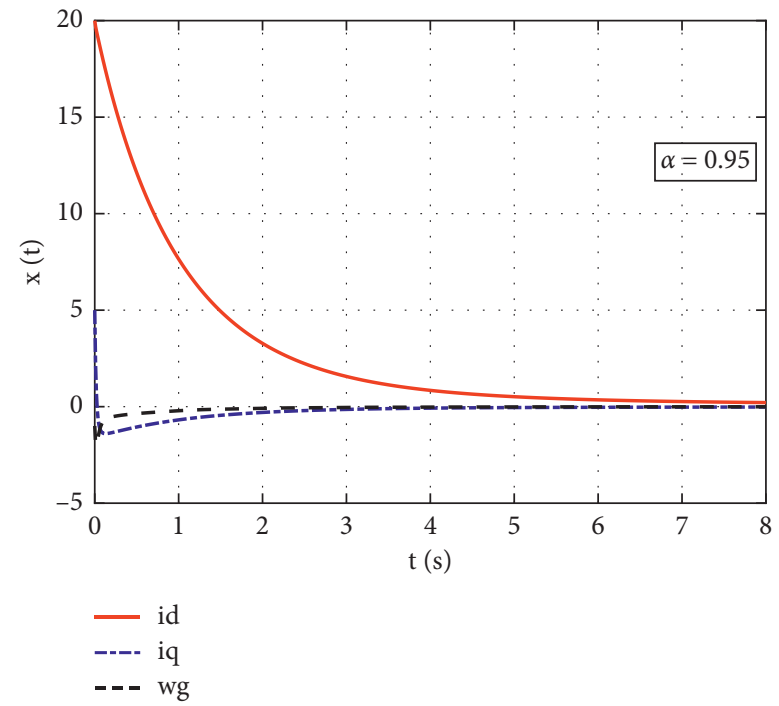

(d)

Figure 5: The fuzzy control system state diagram. (a) $\alpha=1$. (b) $\alpha=0.97$. (c) $\alpha=0.96$. (d) $\alpha=0.95$.

The control quantity is applied to the rotor speed $\widetilde{w}_{g}$ and $q$-axis current $\widetilde{i}_{q}$. Then, we choose the control input matrix $\mathrm{B}_{1}=\mathrm{B}_{2}=[0 ; 1 ; 1]$, and $\Delta B_{1}=\Delta B_{2}=\left[\begin{array}{l}0 \\ 0 \\ 0\end{array}\right], D_{B 1}=D_{B 2}=$ $\left[\begin{array}{lll}0 & 0 & 0 \\ 0 & 0 & 0 \\ 0 & 0 & 0\end{array}\right]$, and $E_{B 1}=E_{B 2}=\left[\begin{array}{l}0 \\ 0 \\ 0\end{array}\right]$.

From Theorem 1, the corresponding control gain matrixes are solved through the YALMIP-MATLAB control toolbox. $K_{1}=[1.3044,52.4198,33.8331]$ and $K_{2}=[7.5833$, $50.3629,32.5048]$.

It can be seen from Figures 5 and 6 that, according to Theorem 1, Ho robust control is applied to the chaotic model of the FOD-PMSG with parameter uncertainties and external disturbance so that the D-PMSG can be stabilized quickly. The anti-interference control performance and robust performance are also improved. With the decrease of the fractional order, Figure 6 shows that the control effect is better. 


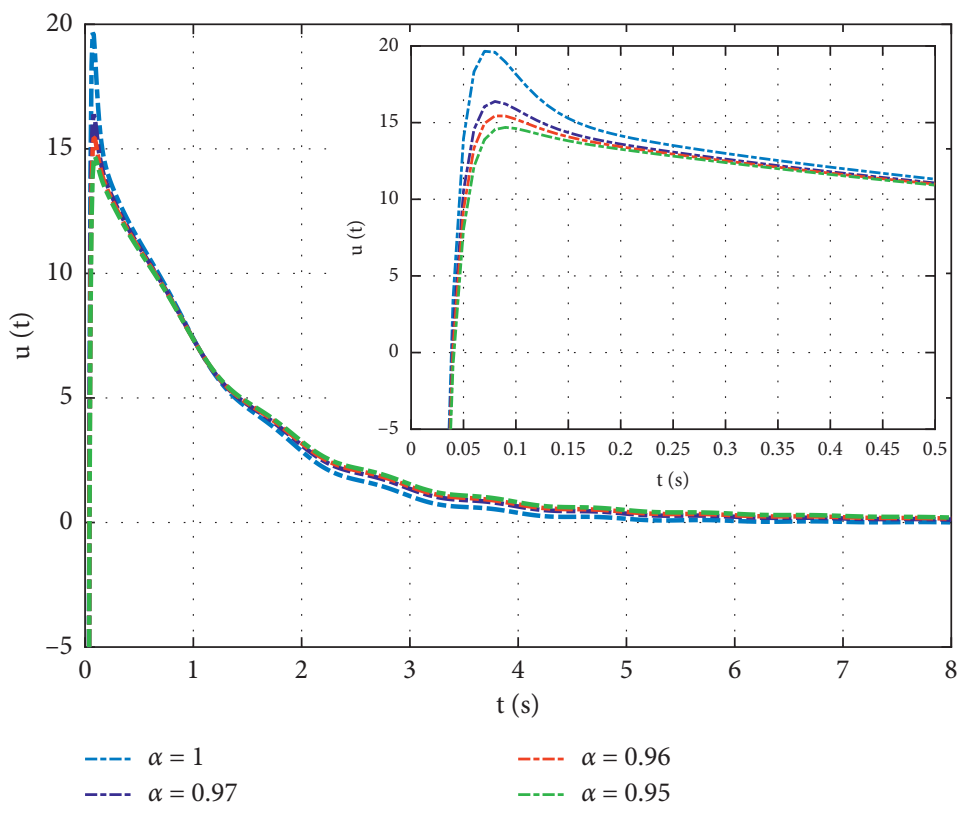

FIgURE 6: The system control curve.

\section{Conclusion}

This paper investigates the stabilization problem of the fractional-order D-PMSG model with parameter uncertainty and external disturbance. Based on T-S fuzzy membership rules, the nonlinear D-PMSG model is equivalently transformed into linear submodels. A new fuzzy state feedback Ho robust control scheme has been proposed for the fractional-order D-PMSG by using the fuzzy PDC technology and LMI approach. By applying a fractional-order Lyapunov direct method, the sufficient conditions for Mittag-Leffler stability satisfying the Ho performance index are derived. In the case of parameter uncertainty and random disturbance, the simulation results show that the designed controller has strong robustness and validate the performance of the proposed fractionalorder control over the integer order. The fractional-order characteristics of the D-PMSG also have a great impact on the control performance. To improve the flexibility of controller design and reduce the conservatism of stability analysis, developing a fuzzy state feedback controller under the imperfect premise matching (IPM) technique for the fractional-order D-PMSG with time delay is one of our future works.

\section{Data Availability}

No data were used to support this study.

\section{Conflicts of Interest}

The authors declare that they have no conflicts of interest regarding the publication of this research article.

\section{Acknowledgments}

This work was supported by the National Natural Science Foundation of China (Grant no. 51907128) and Deyang Science and Technology Plan Project (2019SZ80).

\section{References}

[1] J. Yao, R. Liu, T. Zhou, W. Hu, and Z. Chen, "Coordinated control strategy for hybrid wind farms with DFIG-based and PMSG-based wind farms during network unbalance," Renewable Energy, vol. 105, pp. 748-763, 2017.

[2] C. N. Wang, W. C. Lin, and X. K. Le, "Modelling of a PMSG wind turbine with autonomous control"' Mathematical Problems in Engineering, vol. 2014, Article ID 856173, 10 pages, 2014.

[3] Z. Li, J. B. Park, Y. H. Joo, B. Zhang, and G. R. Chen, "Bifurcations and chaos in a permanent-magnet synchronous motor," IEEE Transactions on Circuits and Systems I: Fundamental Theory and Applications, vol. 49, no. 3, pp. 383-387, 2002.

[4] J. Yu, Y. Ma, H. Yu, and C. Lin, "Reduced-order observerbased adaptive fuzzy tracking control for chaotic permanent magnet synchronous motors," Neurocomputing, vol. 214, no. 19, pp. 201-209, 2016.

[5] G. L. Li Hui-Guang and H. G. Li, "Sliding mode variablestructure control of chaos in direct-driven permanent magnet synchronous generators for wind turbines," Acta Physica Sinica, vol. 58, no. 11, pp. 7552-7557, 2009.

[6] Z. Q. Wu, W. J. Jia, L. R. Zhao, and C. H. Wu, "Maximum wind power tracking for PMSG chaos systems-ADHDP method," Applied Soft Computing, vol. 36, pp. 204-209, 2015.

[7] N. Cao, W. X. Shi, and H. Zhu, "Simulated analysis of dynamic characteristics of permanent magnet direct-drive synchronous wind turbine generators," Electric Machines and Control, vol. 44, no. 1, pp. 104-109, 2017. 
[8] L. Yang, T. Huang, L. Deng, Y. Zeng, and S. Huang, "Analysis on chaotic mechanism of direct-drive permanent magnet synchronous generators based on Lyapunov stability theory," European Journal of Engineering Education, vol. 21, no. 6, pp. 531-537, 2019.

[9] J. H. Park, B. Y. Zhang, and X. N. Song, "Observer-based adaptive hybrid fuzzy resilient control for fractional-order nonlinear systems with-varying delays and actuator failures," IEEE Transactions on Fuzzy Systems, vol. 29, no. 3, pp. 471485, 2019.

[10] J. H. Park, B. Y. Zhang, X. N. Song, and Z. Q. Zhang, "Adaptive command filtered neuro-fuzzy control design for fractional-order nonlinear systems with unknown control directions and input quantization," IEEE Transactions on Systems, Man, and Cybernetics: Systems, vol. 2020, p. 12, 2020.

[11] A. Tepljakov, Fractional-order Modeling and Control Toolbox, Springer, Cham, Switzerland, 2017.

[12] A. Atangana and A. Shafiq, "Differential and integral operators with constant fractional order and variable fractional dimension," Chaos, Solitons \& Fractals, vol. 127, pp. 226-243, 2019.

[13] W. Zheng, Y. Luo, Y. Chen, and Y. Pi, "Fractional-order modeling of permanent magnet synchronous motor speed servo system," Journal of Vibration and Control, vol. 22, no. 9, pp. 2255-2280, 2016.

[14] I. Dimeas, I. Petras, and C. Psychalinos, "New analog implementation technique for fractional-order controller: a DC motor control," AEU-International Journal of Electronics and Communications, vol. 78, pp. 192-200, 2017.

[15] S. P. Nangrani and S. S. Bhat, "Fractional order controller for controlling power system dynamic behavior," Asian Journal of Control, vol. 20, no. 1, pp. 403-414, 2018.

[16] W. Yu, Y. Luo, and Y. Pi, "Fractional order modeling and control for permanent magnet synchronous motor velocity servo system," Mechatronics, vol. 23, no. 7, pp. 813-820, 2013.

[17] W. Xue, Y. Li, S. Cang, H. Jia, and Z. Wang, "Chaotic behavior and circuit implementation of a fractional-order permanent magnet synchronous motor model," Journal of the Franklin Institute, vol. 352, no. 7, pp. 2887-2898, 2015.

[18] T. Aounallah, N. Essounbouli, A. Hamzaoui, and F. Bouchafaa, "Algorithm on fuzzy adaptive backstepping control of fractional order for doubly-fed induction generators," IET Renewable Power Generation, vol. 12, no. 8, pp. 962-967, 2018.

[19] T. K. Mahmoud, Z. Y. Dong, and J. Ma, "A developed integrated scheme based approach for wind turbine," IEEE Transactions on Sustainable Energy, vol. 133, pp. 620-635, 2019.

[20] J. Chen, W. Yao, C.-K. Zhang, Y. Ren, and L. Jiang, "Design of robust MPPT controller for grid-connected PMSG-Based wind turbine via perturbation observation based nonlinear adaptive control," Renewable Energy, vol. 134, pp. 478-495, 2019.

[21] L. Pan and C. Shao, "Wind energy conversion systems analysis of PMSG on offshore wind turbine using improved SMC and extended state observer," Renewable Energy, vol. 161, pp. 149-161, 2020.

[22] K. Tanaka and M. Sugeno, "Stability analysis and design of fuzzy control systems," Fuzzy Sets and Systems, vol. 45, no. 2, pp. 135-156, 1992.

[23] J. Cheng, W. T. Huang, J. H. Park, and J. D. Cao, "A hierarchical structure approach to finite-time filter design for fuzzy Markov switching systems with deception attacks,"
IEEE Transactions on Cybernetics, vol. 2021, Article ID 33502990, 11 pages, 2021.

[24] J. Cheng, H. C. Yan, J. H. Park, and G. D. Zong, "Outputfeedback control for fuzzy singularly perturbed systems: a nonhomogeneous stochastic communication protocol approach," IEEE Transactions on Cybernetics, vol. 2021, Article ID 34236985, 12 pages, 2021.

[25] Y. Li and J. Li, "Stability analysis of fractional order systems based on T-S fuzzy model with the fractional order ( (alpha: $0<\backslash$ alpha <1\)," Nonlinear Dynamics, vol. 78, no. 4, pp. 2909-2919, 2014.

[26] J. Cheng, Y. N. Shan, J. D. Cao, and J. H. Park, "Nonstationary control for T-S fuzzy markovian switching systems with variable quantization density," IEEE Transactions on Fuzzy Systems, vol. 29, no. 6, pp. 1375-1385, 2020.

[27] H. Liu, Y. P. Pan, J. D. Cao, Y. Zhou, and H. X. Wang, "Positivity and stability analysis for fractional-order delayed systems: a T-S fuzzy model approach," IEEE Transactions on Fuzzy Systems, vol. 29, no. 4, pp. 927-939, 2020.

[28] A. Nasiri, S. K. Nguang, A. Swain, and D. J. Almakhles, "Reducing conservatism in an $\$ \mathrm{H}_{-}\{$\infty \}\$ robust statefeedback control design of T-S fuzzy systems: a nonmonotonic approach," IEEE Transactions on Fuzzy Systems, vol. 26, no. 1, pp. 386-390, 2018.

[29] S. Hwang, J. B. Park, and Y. H. Joo, "Disturbance observerbased integral fuzzy sliding-mode control and its application to wind turbine system," IET Control Theory \& Applications, vol. 13, no. 12, pp. 1891-1900, 2019.

[30] B. Herissi and D. Boudjehem, "Fractional-order fuzzy controller for a PMSG wind turbine system," International Journal of Systems Science, vol. 51, no. 16, pp. 3237-3250, 2020.

[31] N. Tidjani and A. Guessoum, "Augmented robust T-S fuzzy control based PMSG wind turbine improved with Ho performance," International Journal of Power Electronics and Drive Systems, vol. 12, no. 1, pp. 585-596, 2021.

[32] G. Si, J. Zhu, L. Diao, and Z. Ding, "Modeling, nonlinear dynamic analysis and control of fractional PMSG of wind turbine," Nonlinear Dynamics, vol. 88, no. 2, pp. 985-1000, 2017.

[33] M. Borah and B. K. Roy, "Dynamics of the fractional-order chaotic PMSG, its stabilization using predictive control and circuit validation," IET Electric Power Applications, vol. 88, pp. 985-100, 2017.

[34] P. Mani, R. Rajan, L. Shanmugam, and Y. H. Joo, "Adaptive fractional fuzzy integral sliding mode control for PMSM model," IEEE Transactions on Fuzzy Systems, vol. 27, no. 8, pp. 1674-1686, 2019.

[35] P. Mani, R. Rajan, and Y. H. Joo, "Design of observer-based event-triggered fuzzy ISMC for T-S fuzzy model and its application to PMSG," IEEE Transactions on Systems, Man, and Cybernetics: Systems, vol. 51, no. 4, pp. 2221-2231, 2021.

[36] D. C. Huong and M. V. Thuan, "Mixed \$H_\{linfty\} and passive control for fractional-order nonlinear systems via LMI approach," Acta Applicandae Mathematica, vol. 170, no. 1, pp. 37-52, 2020.

[37] R. E. Gutiérrez, J. M. Rosário, and J. T. Machado, "Fractional order calculus: basic concepts and engineering applications," Mathematical Problems in Engineering, vol. 2010, Article ID 375858, 20 pages, 2010.

[38] X. N. Song, Control and Synchronization of Fractional Order Chaotic Systems, Science Press, Beijing, China, 2018. 
[39] R. Caponetto, G. Dongola, and L. Fortuna, Fractional Order Systems: Modeling and Control Applications, World Scientific Publishing, Singapore, 2010.

[40] Y. Li, Y. Chen, and I. Podlubny, "Stability of fractional-order nonlinear dynamic systems: Lyapunov direct method and generalized Mittag-Leffler stability," Computers \& Mathematics with Applications, vol. 59, no. 5, pp. 1810-1821, 2010.

[41] S. Zhang, Y. Yu, and J. Yu, "LMI conditions for global stability of fractional-order neural networks," IEEE Transactions on Neural Networks and Learning Systems, vol. 28, no. 10, pp. 2423-2433, 2017.

[42] L. Xie, "Output feedback Ho control of systems with parameter uncertainty," International Journal of Control, vol. 63, no. 4, pp. 741-750, 1996.

[43] S. Boyd, L. E. Ghaoui, E. Freon, and V. Balakrishnan, Linear Matrix Inequalities in System and Control Theory, SIAM, Philadelphia, PA, USA, 1994.

[44] M. S. Tavazoei and M. Haeri, "Chaotic attractors in incommensurate fractional order systems," Physica D: Nonlinear Phenomena, vol. 237, no. 20, pp. 2628-2637, 2008.

[45] L. Yang, T. M. Huang, L. Deng, Y. F. Zeng, and S. D. Huang, "Adaptive sliding mode control of chaotic motion in directdrive permanent magnet synchronous for wind turbines," Acta Microscopica, vol. 29, no. 2, pp. 658-665, 2020.

[46] Z.-H. Xiu and G. Ren, "Stability analysis and systematic design of Takagi-Sugeno fuzzy control systems," Fuzzy Sets and Systems, vol. 151, no. 1, pp. 119-138, 2005. 